\title{
Contribution to the Miniaturization of Antennas: State of the Art
}

\author{
Mohamed Tarbouch ${ }^{1}$, Abdelkebir El Amri ${ }^{2}$ and Hanae Terchoune ${ }^{3}$ \\ RITM Laboratory, ESTC, Hassan II University, CASABLANCA, MOROCCO \\ ${ }^{1}$ mtarbouch@gmail.com, ${ }^{2}$ elamri_abdelkebir@yahoo.fr, ${ }^{3}$ hanae.terchoune@gmail.com
}

\begin{abstract}
Nowadays, communications systems have become more mobile and personal, that means materials used must be as smaller as possible. Low weight reduced volume and low cost are some of the characteristics that must be accomplished in modern telecommunication equipment. It is well known that the antenna is one of the most important devices in the radiofrequency (RF) section. This element needs to perform effectively according to the necessities. To achieve low weight, small volume and low cost, the size needs to be reduced, but keeping the main characteristics. The size reduction of antennas can be made by several techniques according to the type of the element. In this study we will present the fundamental limitations of small antennas as a major constraint of antenna miniaturization, then we will discuss major techniques that have been reported in literature to reduce the size of an antenna. Feasibility, features, effects on the miniaturisation of antennas of each technique will be presented and discussed. At the end of this study we will give some examples of real systems that used antennas miniaturization techniques.
\end{abstract}

Keywords: Antennas Miniaturization techniques, Small Antennas, wireless communications.

\section{Introduction}

Many of new wireless communications devices such as Smart-phones, tablets, Laptop, and other embedded systems use one or multiple frequency band, the standardized band are defined in the following frequency range: Long Term Evolution LTE (698 MHz - $806 \mathrm{MHz}$ ), Global System for Mobile Communications GSM 850/900 (824 MHz - 894 MHz / 880 MHz - 960 MHz), Personal Communication Services PCS 1900 (1850 MHz - 1990 MHz), Digital Communication System DCS 1800 (1710 MHz - 1880 MHz), Universal Mobile Telecommunications Systems UMTS (1920 MHz - $2170 \mathrm{MHz}$ ), 3G (1710 MHz $1755 \mathrm{MHz})$ and 4G (2110MHz - $2155 \mathrm{MHz} / 2500 \mathrm{MHz}-2700 \mathrm{MHz})$. Conventional antennas for Long Term Evolution LTE band as a lower band have large physical sizes, that is, $21.5 \mathrm{~cm}$ which is not a practical length. To allow more attractive and practical products or simply to reduce costs, antenna engineers are routinely asked to reduce the antennas size without significantly affecting their performance.

In the literature we have not found a comprehensive study that treated all of the antenna miniaturization techniques, all what we found are studies that treated a type of very specific antenna or studies that processes a single technique. This study includes virtually all antenna miniaturization techniques.

This study will be a great starting point for students and researchers interesting to the field of antennas miniaturization. At the end of each chapter dealing with a technique, the reader will find a summary of 
this technique as well as the advantages and disadvantages resulting from this technique, and will accordingly choose the technique that suits him.

\section{Fundamental Limits of Antenna Size}

It has long been recognized that, for a given frequency, the size of an antenna cannot be indefinitely reduced without compromising the $Q$ (quality factor), the efficiency or the gain. This problem was first explored in the 1940s. In 1947, Wheeler [1] derived a relation between antenna volume and the maximum achievable 'power factor' (equal to the inverse of the quality factor). He demonstrated that reducing the antenna size results in narrow bandwidth.

In 1948, Chu [2] extended Wheeler's analysis by expressing radiated fields in terms of spherical modes. Chu's method is general and can be applied to any antenna; His work led to the same conclusion as Wheeler.

A second phase of work on the limits of small antennas occurred in the 1960s. In 1960, Harrington [3] related antenna size, minimum $Q$ and gain (including losses) for linearly and circularly polarized waves. In 1964, Collin and Rothschild [4] presented an exact method for finding the minimum Q without using the approximate equivalent network of Chu, for both spherical and cylindrical modes. In 1969, Fante [5] extended these results to include antennas with mixed polarization. Later, McLean $[6,7]$ presented a simple but exact method by determining the minimum antenna $\mathrm{Q}$.

From all of the efforts that have been listed above, as well as others, we know that the minimum unloaded

$Q_{0}$ at resonance of a linearly polarized antenna (when a single lowest order TM mode is implied) is given by:

$$
Q_{0}=\eta_{A}\left(\frac{1}{(\beta r)^{3}}+\frac{1}{\beta r}\right)
$$

Where $r$ is the minimum radius of a sphere that just encloses the antenna, $\beta$ is the wave number $(2 \pi / \lambda)$ and $\eta_{A}$ is the antenna efficiency. It should be noted here, that the antenna may be brought to resonance using an external component. Should this be the case, the $Q$ in Equation (1) is that of the antenna and component combined. An antenna is often considered to be electrically small when the following condition is met :

$$
\frac{r}{\lambda} \leq \frac{1}{2 \pi}
$$

From Equation (2), for small antennas $\beta r$ is less than unity and the $1 / \beta r$ term is small compared to $\frac{1}{(\beta r)^{3}}$ ; therfore, Equation (2) can be approximated by:

$$
Q_{0} \approx \eta_{A}\left(\frac{1}{(\beta r)^{3}}\right)
$$

Since the volume of the sphere containing the antenna is proportional to the cube of its radius, the unloaded quality factor is proportional to efficiency and inversely proportional to volume. This formula 
(3) is realistic and it's clear from it that small antennas exhibit a trade-off between size, $Q$ factor (bandwidth) and efficiency.

In the following part we will present and discuss main techniques reported in the literature for antenna miniaturisation. These miniaturisation techniques include reactive loading, material loading, reshaping the antenna geometry, shorting and folding, modification of ground plane, top loading and the use of metamaterials.

\section{Main Techniques for Antenna Miniaturisation}

\subsection{Reactive Loading}

Reactive loading is a common method to reduce the resonant frequency of a small antenna. The aim is to store enough magnetic energy (to create inductance) to counter the electric energy (capacitance) that is associated with most small antennas, this is illustrated for two meandered structures in Figure1.

The bold arrows show, broadly speaking, where radiation occurs. The non-bold arrows can be considered to be in pairs that have opposite directions. Hence, they cancel in the far field and do not produce any radiation. However, they do store energy in the near field, and this can be used to reduce the antenna resonant frequency.

An alternative way of viewing this is that the antenna length is maintained at approximately $\lambda / 4$, so that the resonant frequency is the same as that of an equivalent $\lambda / 4$ monopole. However, since radiation comes from less of the structure, the radiation resistance must be reduced. In practice, the length of the meander must be somewhat longer than $\lambda / 4$ in order to achieve resonance.
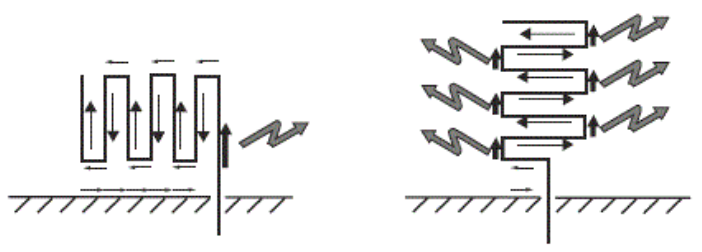

Figure1: Radiation from meandered structures

Depending to antenna type studied there are some form of reactive loading.

In [8] a small-sized, dual-port slot antenna has been developed for use with contact free ID cards at 2.45 $\mathrm{GHz}$. By using a loading capacitor, the length of the slot antenna can be reduced by approximately $17 \%$.The measured gain of the antenna is approximately $4.8 \mathrm{dBi}$.

In [9] as shown in Figure2 new structures of slot antenna with capacitive loading and inductive loading simultaneously have been presented. One of designs has $52.9 \%$ Size reduction of slot length compared to the conventional slot antenna and $2.8 \%$ impedance bandwidth, new structures have same radiation pattern as the conventional slot antenna. 


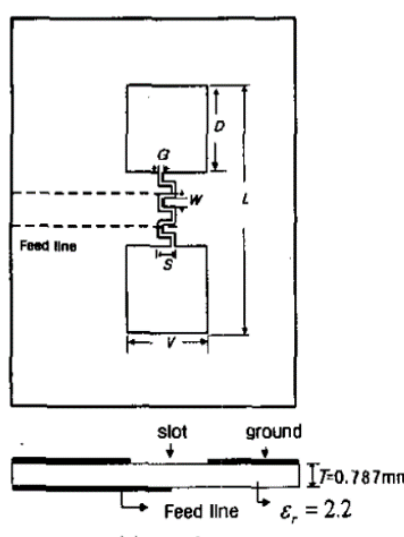

(a) type A

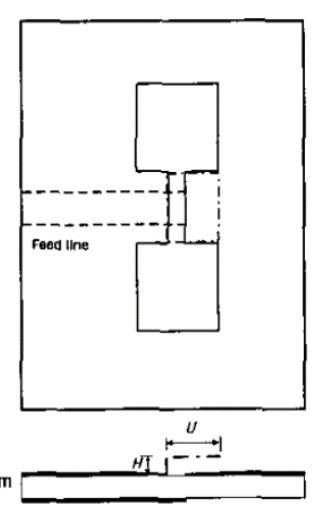

(b) type B

Figure 2: Microstrip-fed slot antenna by inductive and capacitive loading

In [10] a square patch antenna with notches cut into two of the sides. The notches provide a reactive loading allowing the use of thick substrate $\left(\approx 0.08 \lambda_{0}\right)$ and induce a second resonance near the main patch resonance. The antenna has an area that is $94 \%$ smaller than the area occupied by a square half wave patch and maintains a bandwidth of $13.2 \%$. However many important antenna parameters were not studied in this paper.

Many works have appeared in the literatures which take about size reducing of wire antennas type using reactive loading, it is known that the input impedance of a half-wavelength dipole is capacitive below the resonance frequency. Any attempt to reduce the resonant frequency of the dipole antenna must therefore add an inductive load to the dipole in such a manner so that the capacitive impedance of the dipole is exactly cancelled at the reduced resonant frequency, in [11], miniaturization of a dipole antenna is achieved using a loop as a reactive loading element as show in Figure3. It is seen that the reactive loading reduces the resonant frequency of the dipole antenna to bring it very close to the electrically small antenna limit without affecting the radiation pattern, the configuration reduces the resonant frequency of the dipole by $26.01 \%$.

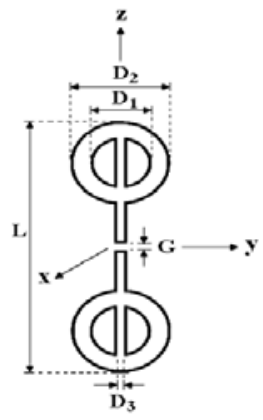

Figure 3: Double-loop loaded dipole antenna

Many other works have also appeared in the literature which followed approaches that are similar to the one mentioned previously for dipole antenna [12-14]. In [12] a folded monopole antenna with LC-loadings is designed for a miniaturized antenna. The antenna can be miniaturized because the operating frequency of the designed antenna is essentially determined by the distributed inductors and capacitor on the folded monopole antenna. The antenna was fabricated as shown in Figure4 


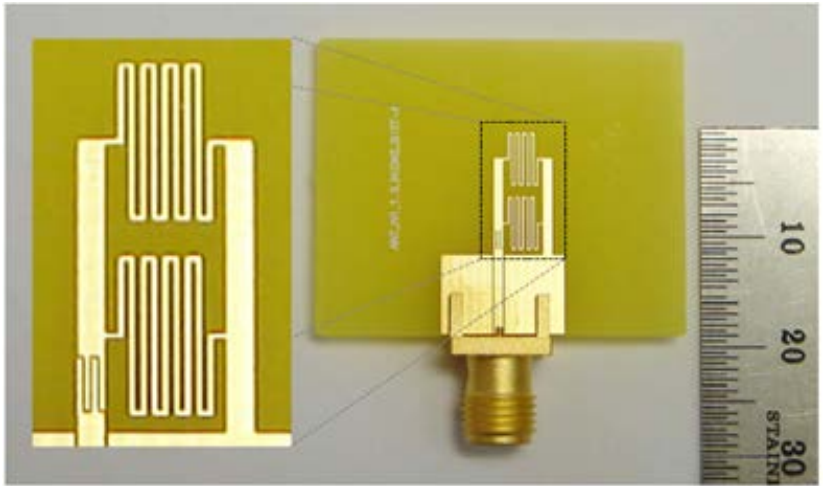

Figure 4 : Compact Folded Monopole Antenna with LC-Loadings

The length of the radiator of the designed antenna is only $11.05 \mathrm{~mm}$, which corresponds to $0.077^{\lambda_{0}}$. It was found that the ground plane size did not affect the characteristics of the proposed antenna. Indeed It was shown that changing the ground plane with various widths results in all resonant frequencies of the antennas are within $2.09 \mathrm{GHz} \sim 2.16 \mathrm{GHz}$, and bandwidths are about $90 \mathrm{MHz}$ in all the cases, The maximum total gain of the antenna is $-2.01 \mathrm{dBi}$ at the resonant frequency of $2.095 \mathrm{GHz}$.

In [13], as shown in Figure5 a Yagi-Uda antenna is designed and built, employing cylindrical-hat covers in all elements to load inductively each part of the structure. With this proposal, the effective area of the antenna is diminished almost $35 \%$, preserving the bandwidth.

The main disadvantage obtained with this technique, is the small reduction of the gain, which is decreased almost $1.3 \mathrm{~dB}$.

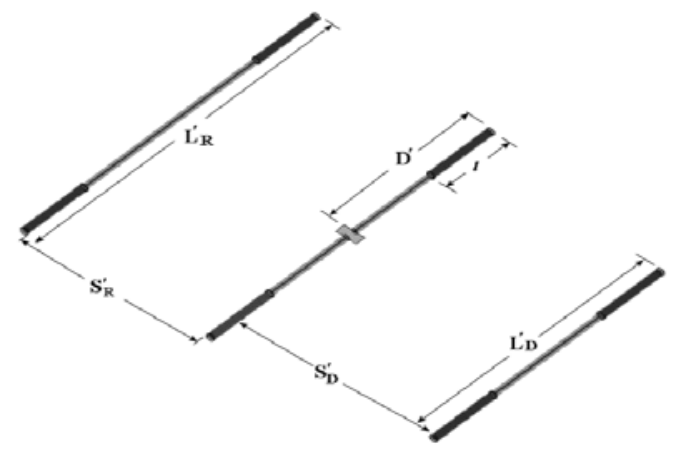

Figure 5: Inductively-Loaded Yagi-Uda Antenna

In [14], as shown in Figure6 a log-periodic dipole antenna loaded with distributed component, operating between $8 \mathrm{GHz}$ and $18 \mathrm{GHz}$, has been designed. By adding the C-shaped stub, the dimension can be miniaturized by $60 \%$ compared with conventional LPDA, but the radiation performance of the antenna suffers some influence, although, the reflection coefficient is beyond $-10 \mathrm{~dB}$ within a narrow band. 


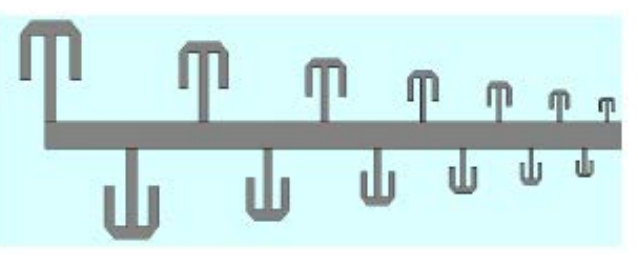

Figure 6 : Printed Log-periodic Antenna with Loaded Stub

Most popular antennas can therefore be miniaturized using reactance loading. Many types of methods can be used to fabricate reactance: making slots, notches or loops, and in some cases materials with high dielectric permittivity are required.

However, this method lacks a general MOP (Method of procedure) and depends on type of antenna in hand; also in some cases this method comes at a cost of small reduction of the gain and limited bandwidth.

\subsection{Material Loading}

Antennas with dielectric loading have the advantage that tuning capacitance can easily be built in to the structure. A high dielectric constant material can be used to achieve a slow wave structure that allows smaller resonators to be realized.

Various studies have investigated different materials as well as configurations to effectively use the above approach to miniaturize a Microstrip Patch Antenna (MPA). [15] reports all of the major and novel techniques, which have appeared in the literature related to micro strip antenna miniaturisation, in this part we will report two cases studied in [15] related to MPA dielectric loading and we will report also others works and studies that appeared in the literature which investigated dielectric loading for other popular antenna types.

In [16], several MPA designs were investigated using various types of ceramic substrates. A square MPA was fabricated on a low-temperature co-fired ceramic (LTCC) substrate of $\varepsilon r=100$. A reduction of the area of the patch by a factor of 8 was achieved as compared with that of a conventional patch printed on an FR4 substrate. However, the substrate was chosen to be relatively thick $(0.031 \lambda)$ to mitigate the problem of low bandwidth. The antenna had a fractional bandwidth of $7.2 \%$ and a gain of $2.8 \mathrm{dBi}$ at $1.88 \mathrm{GHz}$. Another MPA was fabricated using a textured ceramic substrate, which was a mixture of LTCC and Stycast, and resulted in an effective permittivity of 23.5. The MPA printed on this substrate was 2.5 times smaller than the conventional MPA fabricated on an FR4 substrate. The measured gain of the antenna was $3 \mathrm{dBi}$ and it had a fractional bandwidth of $9.1 \%$ centred about $1.7 \mathrm{GHz}$. The bandwidth of the MPA was also found to improve, albeit at the expense of its gain, using a bow-tie shape. Thus, through various design choices, significant miniaturisation was achieved using ceramic substrates while maintaining good antenna gain as well as bandwidth.

MPA miniaturization using a magnetodielectric substrate has been analyzed in details in [17]. A magnetodielectic substrate design was presented in [18]. The MPA fabricated on the proposed substrate was found to be $65 \%$ smaller, as compared with a conventional patch resonating at $2.45 \mathrm{GHz}$. The MPA had a fractional bandwidth of $0.5 \%$ and radiation efficiency was $45 \%$.

In [19] effect of loading a simple monopole antenna shown in Figure7 with magneto-dielectric material is investigated, Material loading of monopole antennas with magneto-dielectric materials have an advantage of higher bandwidth compared to loading with magnetic materials of equal refractive index, a 
Mohamed Tarbouch, Abdelkebir El Amri and Hanae Terchoune; Contribution to the Miniaturization of Antennas: State of the Art, Transactions on Networks and Communications, Volume 4 No. 5, October (2016); pp:48-77

significant downward shift in resonant frequency is observed and an efficiency of around $60 \%$ is obtained using ferrite ceramic based magneto-dielectric material properties « $\varepsilon r=3.75, \mu r=2.5 »$. Nevertheless, this is no insight into gain characteristic.

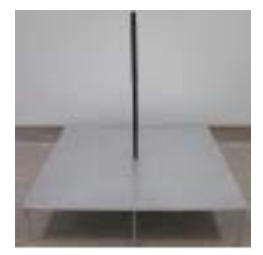

Figure 7: Monopole antenna

In [20] as shown in Figure8 a Vee dipole antennas is studied, The study starts with designing a Vee dipole antenna with a $44 \mathrm{~mm}$ arm length and a UWB coaxial balun, To achieve a small scale version of this Vee dipole antenna, both the arm and balun length are reduced in size, it is shown that the reduction in the Vee arm length affects the lower frequency of operation, coaxial cable size made it possible to reduce the balun length without affecting the matching of the Vee dipole antenna.

To compensate for the reduction in Vee arm length, a dielectric cone with $\varepsilon r=6$ is implemented between the Vee dipole arms, so a small reduction in the lower frequency end from 3.2 to $2.95 \mathrm{GHz}$ is achieved. The new antenna with the dielectric hollow cone has higher gains at all frequencies and still having same radiation characteristics.

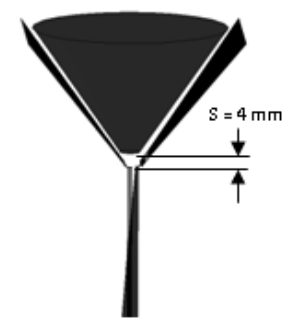

Figure 8: V dipole Antenna

In [21] a miniaturized monofilar helical antenna using dielectric loading was presented. The height (and other dimensions) of the antenna is reduced by applying a hemispherically shaped dielectric material of high dielectric constant above the ground plane and around the helix. The dielectric constant of the loading material was arbitrarily chosen to be 9. Next, the wave impedance mismatch at the outer boundary of the dielectric hemisphere loading this miniaturized helical antenna is addressed creating a spatial matching network. A simple quarter wave transformer is chosen for this task and is implemented by adding a second layer of dielectric material around the hemisphere with dielectric constant $\varepsilon r=3$. When compared with an unloaded antenna design, the total height of the antenna has been reduced by $46.8 \%$ while maintaining the wide input impedance bandwidth (i.e., 48.0\%), the gain pattern stability over frequency, and the required axial ratio for circular polarization over the entire passband. All of these characteristics were achieved while maintaining essentially the same lowest frequency of operation as the unminiaturized version of the antenna. The designed antenna is shown in Figure 9 


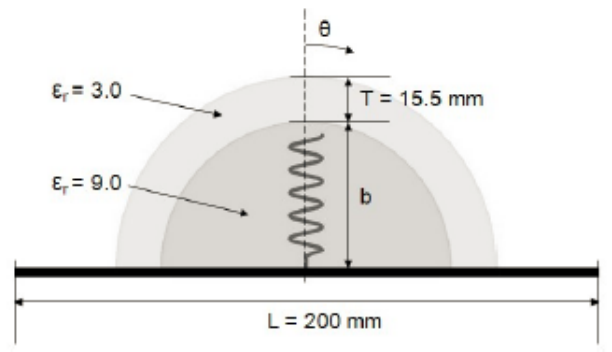

Figure 9: Helic antenna structure

This method is widely used in different antenna type and provides several degrees of miniaturization, this method lacks a general MOP (Method of procedure) and depends on type of antenna. Most of the presented designs based on this method had radiation efficiency of about $45-60 \%$ but keep fixed the lower frequency of operating bandwidth. However, major disadvantages of this method of size-reduction are the cost of such substrates as well as limited obtained bandwidth.

\subsection{Reshaping Antenna Geometry}

\subsubsection{Fractal}

An antenna can also be miniaturized by making changes in the shape of its geometry and its feed line. One of the interesting techniques to provide this kind of antenna is the use of fractal structures, REHA provided in [22] generalities of fractal geometries and their dimensions, after that, he described some linear fractal geometries such as KOCH, SIERPINSKI, DRAGON, TREE, CIRCULAR, CANTOR SET, HILBERT, MINKOWSKI, and finally he discussed the applications of these geometries and their performances in the design of the miniaturized antennas.

In this part, we will present some examples of using Fractal in order to reduce antenna size, as shown in Figure10, [23] presents a compact antenna based on the Hilbert curve fractal. The basic antenna is designed at $2.4 \mathrm{GHz}$, one of the frequencies used in RFID applications, it was shown that the antenna size can be reduced up to ten times in the third iteration and the resonant frequency has shifted down by approximately $0.09 \mathrm{GHz}$ from the first iteration. However this is no insight into others Antenna characteristics.

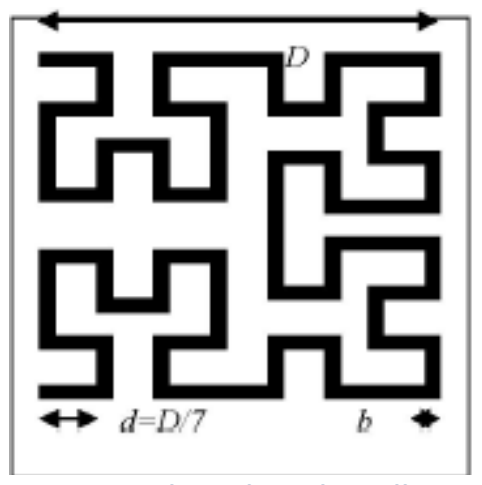

Figure 10: Antenna based on the Hilbert curve fractal

In [24] multi-fractal geometry is introduced into the conventional triangular patch antenna with microstrip feed. The Figure11 shows the Antenna design, after addition of Koch and Sierpinski fractal. 


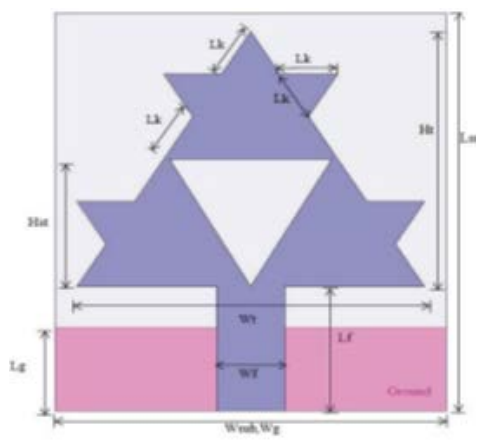

Figure 11: Triangular patch antenna modified by Koch and Sierpinski fractal

The proposed antenna was observed to cover a frequency range of $4.68 \mathrm{GHz}-6.43 \mathrm{GHz}$ with a return loss of $-31.9 \mathrm{~dB}$ at a resonant frequency of $5.48 \mathrm{GHz}$. The resonant frequency is observed to decrease considerably with the addition of each fractal technique there by covering the lower frequencies in the operating band.

As the fractal iterations are done, it is found that the lower cut-off frequency reduces covering the lower frequencies. This effect helps in antenna miniaturization as the antenna covers the frequencies which are lower than its operating frequency range.

In [25] the Koch iteration technique has been applied to a simple folded-slot antenna to obtain first iteration and second iteration fractal versions of the folded-slot antenna, the designed antenna is shown in Figure12. It can be noted that a significant shift in the resonant frequency was observed for the first iteration Koch fractal folded slot antenna when compared to that of a simple folded-slot antenna. However, the resonant frequency did not reduce significantly for the second iteration Koch fractal antenna in spite of the increase in the total length compared to the first iteration version. Therefore, the first iteration of Koch fractal can help to reduce significantly the antenna size. However it was shown that the bandwidth obtained of the Antenna was decreased as the iteration order is increased.

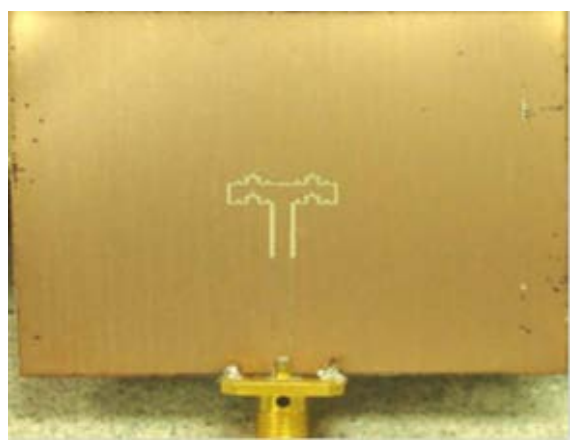

Figure 12: folded-slot antenna modified by Koch fractal

In [26] the author presents the design of fractal patch antenna based on the basic structure of square antenna (Figure13). This one operates at $1.575 \mathrm{GHz}$ for Global Positioning System (GPS) application. The Koch fractal structure was designed with 0.25 iteration factor. The Koch curve removes at the center of each side is $25 \%$ of the side length due to 0.25 iteration factor. It can be seen that in the first iteration the frequency has drops to $1.19 \mathrm{GHz}$ from $1.575 \mathrm{GHz}$. The resonant frequency drops is approximately equal 
to $24 \%$ from the basic structure resonant frequency, from antenna size point of view fractal technique can reduce the size up to $43.2 \%$. The second iteration fractal can reduce the size about 47.5 percent.

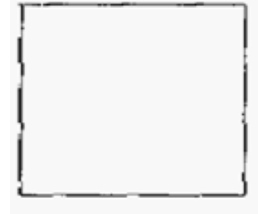

(a)

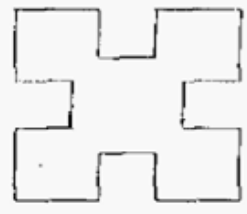

(b)

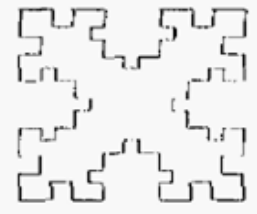

(c)

Figure 13: Antenna structures (a) basic square, (b) first iteration fractal, (c) second iteration fractal

Fractals are space-filling geometries that can be used as antennas to effectively fit long electrical lengths into small areas. Therefore, miniaturized antennas can be fabricated using some generating iterations of the generating procedure. Four applications of these miniaturized elements were demonstrated using patch and slot antenna.

\subsubsection{Adding Truncation: Slots, Notches and Cuts}

This method is widely used to miniaturize the Micro Strip Patch Antenna (MPA), several works [27-39] have appeared in the literature in which the size of the MPA has been reduced by introducing various types of slots in the MPA. The use of this method generally leads to poor polarization purity. However, by symmetrical arrangement of the slots on the patch, such purity can be preserved [27]. A miniaturization of $40-75 \%$ has been achieved by inserting different types of slots in the MPA.

Most of the presented designs based on this method had low radiation efficiency of about $25 \%$ but provided wider operating bandwidths when using slots. Some had a fractional bandwidth of up to $5.5 \%$ [28].

As shown in Figure14, [38] used two techniques: notches inserting to adapt the structure and nicks inserting to reduce the size of the antenna, it establishes a final prototype of a miniature and adapted antenna with a reduction rate of $42.9 \%$ over the length of the reference antenna. However the gain of the antenna is decreased. [39] Proposed a novel design for miniaturized surface-wave holographic antenna, the new structure was obtained by making cuts in perfect magnetic conductor (PMC) boundary to achieve higher gain and smaller dimension, by miniaturization the area of the original antenna is decreased by over $80 \%$. The proposed antenna is shown in Figure15.

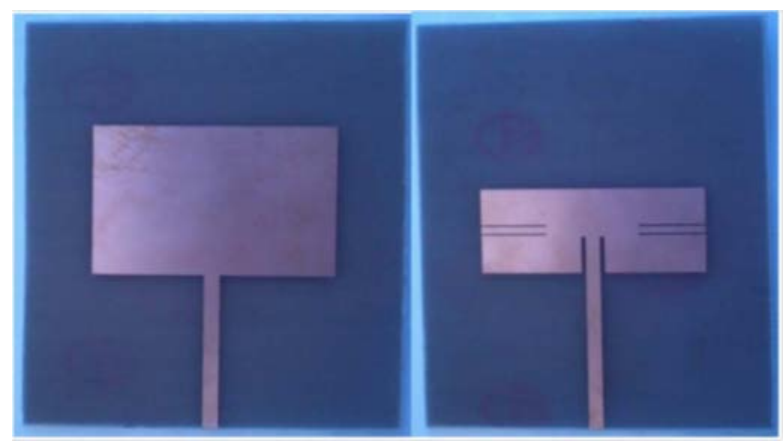

Figure 14: The realized prototypes: (a) The circuit of the reference antenna, (b) The optimized antenna 


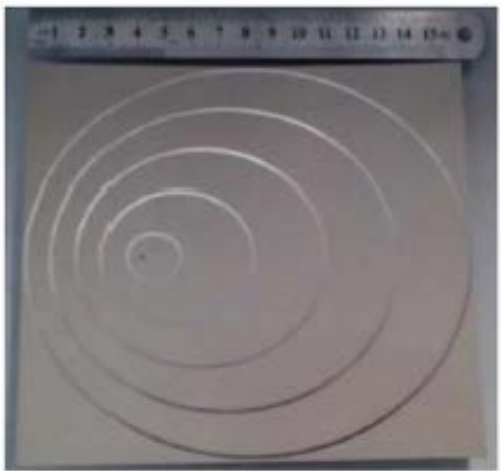

(a)

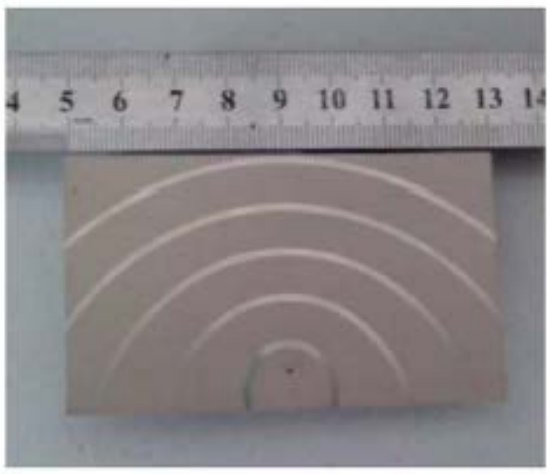

(b)

Figure 15: Antenna Structure (a) the original surface-wave holographic antenna and (b) the proposed miniaturized one

\subsubsection{Antenna-Feed Line Transition}

There are many configurations that can be used to feed a patch antenna. [40] compared the effect of three feeding methods: coaxial probe (Figure16 - case1), Microstrip line with the ground plane in the reverse face of the patch (Figure16 - case2), and Microstrip line with the ground plane in the same face of the patch (Figure16 - case3). It was shown that for the case 3 (Figure16) the resonant frequency is lower than for the case 1 and 2. This is an important advantage to design a miniaturized antenna without increasing its volume and surface. Also it was demonstrated that the bandwidth is larger than those of case 1 and case 2.

In [41] A method to design a microstrip-fed antipodal tapered- slot antenna, which has Ultra Wide Band (UWB) performance and miniaturized dimensions, is presented. The proposed method modifies the antenna's structure to establish a direct connection between the microstrip feeder and the radiator. That modification, which removes the need to use any transitions and/or baluns in the feeding structure, is the first step in the proposed miniaturization. In this step, of the miniaturization process, the structure is modified by removing the tapered ground plane, and the slotline to microstrip transition. A microstrip line is connected directly to the top layer in the manner shown in Figure 17(b), with this modification in the structure, the overall dimensions of the structure we get a reduction in size by $33 \%$.

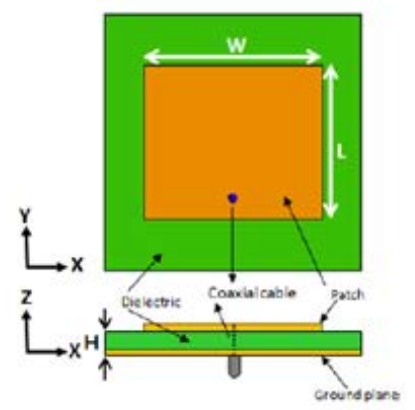

(a) Case 1

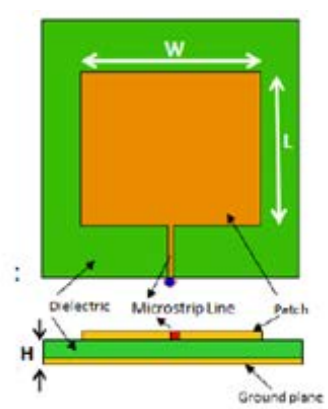

(b) Case2

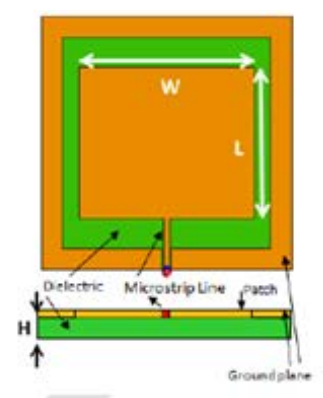

(c) Case 3

Figure16: The different feeding methods 

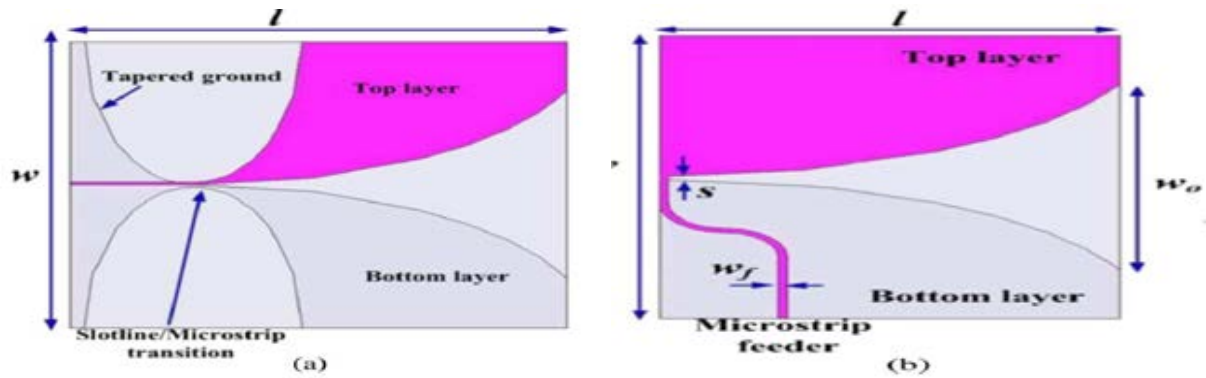

Figure17: Configuration of (a) the traditional antipodal, (b) modified structure

\subsubsection{Use of Genetic Algorithm}

Genetic algorithm (GA) is a powerful optimization technique that has been shown to be useful in a wide area of electromagnetic. GA is applied to design broadband, multiband and miniature antenna. It is a robust, stochastic-based search method, which can handle the common characteristics of electromagnetic optimization problems that are not readily handled by other traditional optimization methods. Many researches studies Antenna can be shaped and optimized to obtain a large electrical length in limited space using the genetic algorithm [42-43].in [42] a printed antenna occupying the same physical volume as a rectangular patch was designed to resonate at a frequency that is $37 \%$ lower than the resonance frequency of the patch. A Genetic Algorithms optimization procedure was used in the design. The proposed antenna has a surface wave efficiency of about $95 \%$, but has high cross polarization level.

In [43] an efficient method which use genetic algorithm to optimize PIF A antenna parameters has been presented. Two examples of optimal antennas are designed with the proposed method. The simulated results show the optimized antennas realize a minimization of reflection coefficient, reduction in size of the antenna and increasing the bandwidth, while maintaining good radiation performances.

Reshaping method is widely used in different designs and provides several degrees of miniaturisation, but a part from Fractal geometry this method lacks a general design methodology. Most of the presented designs based on this method had low radiation efficiency of about $25 \%$ but provided wider operating bandwidths when using slots.

\subsection{Shorting and Folding}

Several works have appeared in the literature that presented miniaturized antenna using the shorting or folding techniques [44-50].

In [44] an electrically small circular microstrip probe-fed patch antenna was designed. As shown in Figure18 a single shorting post is incorporated in the design, resulting in the diameter of the radiating element being significantly reduced. The radius of this modified circular patch is $9.19 \mathrm{~mm}$, about $0.047 \lambda 0$. This compares with a conventional circular patch (mounted on the same substrate and designed to resonate at the same frequency) with a radius of $27.1 \mathrm{~mm}(R=0.14 \lambda 0)$. The significant reduction in size is obtained, however, at the cost of reduced bandwidth, for a conventional circular microstrip patch the predicted $10 \mathrm{~dB}$ return loss bandwidth is $1.8 \%$, compared to $1.2 \%$ for the single shorting post antenna. Also it was found that the predicted directivity of the shorted patch is $2.9 \mathrm{~dB}$ compared to $4.0 \mathrm{~dB}$ for a conventional circular patch engraved on the same substrate. 


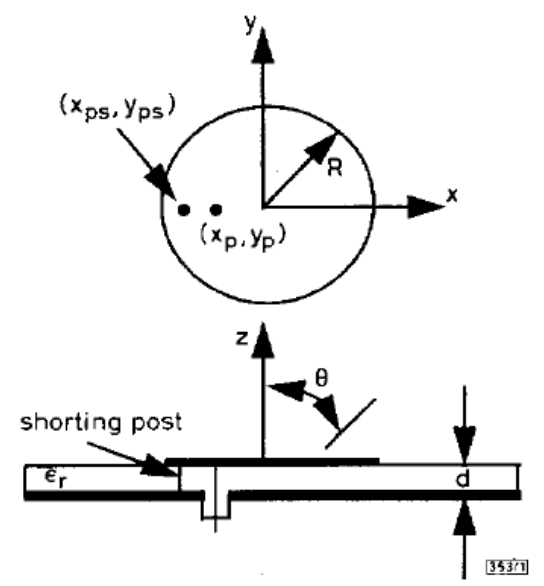

Figure18: Schematic diagram of probe-fed circular microstrip patch antenna with single shorting post

The idea of a folded shorted micro strip patch antenna has been studied in details in [45]. As shown in Figure19 the technique enables a decrease of the original shorted (quarter-wavelength) patch dimension by the factor $1 / \mathrm{N}$ where $\mathrm{N}$ is a number of vertically placed patch plates above the ground plane while maintaining a quarter wavelength resonant length. Using this technique two antennas for $1575 \mathrm{MHz}$ GPS band and $869 \mathrm{MHz}$ RFID band have been designed, the length of these antennas has been reduced to $\lambda 0 / 8.6$ and $\lambda 0 / 11.7$. It was found that this technique has a negative effect on the impedance bandwidth for antenna.

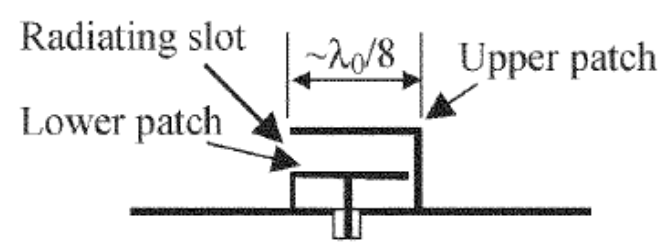

Figure19: $\lambda$ /8 shorted/folded patch antenna

The same technique as has been used in [46] to make a $\lambda$ /8 folded MPA for ISM band (2.4-2.483 GHz). The radiation efficiency of the designed antenna is measured to be approximately $90 \%$ and an impedance bandwidth of $4 \%$.

Folding techniques have been used by many authors to reduce the size of wire antennas, such as helical, loop and monopole antennas [47-50]. They consist of using continuously folded wire antennas, which tend to resonate at much lower frequencies than an ordinary antenna of equal length.

In [47] a new Spiral-Folded, Printed Quadrifilar Helical Antenna (S-FPQHA) design is presented. It provides a 43\% reduction in size for the axial length of the antenna. The planar unwrapped representation of the S-FPQHA, as well as the conventional PQHA, is illustrated in Figure20. The four arms of these antennas were printed onto a thin dielectric substrate wrapped around a cylindrical support, although, the design results in a slight reduction in efficiency. 


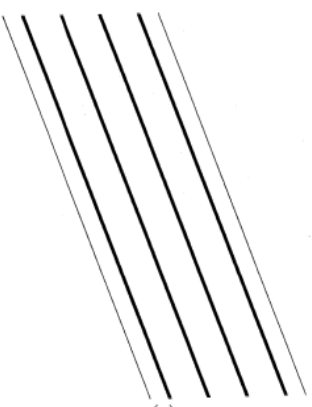

(a)

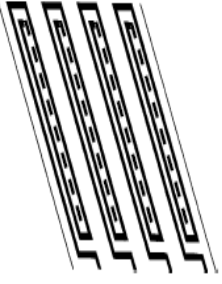

(b)

Figure20: the unwrapped PQHA (a) S-FPQHA (b)

In [48] a theoretical model has been presented for electrically short antenna, different half-wave winding resonant structures has been presented, finally conditions for electrically small antenna have been demonstrated.

In [49] a meander wire antennas has been introduced with a size reduction in the resonant length typically from $25-40 \%$. The efficiency is affected only by the ohmic losses in the wire and cross polarization is negligible. More size reduction is obtained by decreasing the radius of the wire or increasing the separation of the folded arms. The reduction in size is obtained, however, at the cost of reduced bandwidth.

In [50] two types of modified dipole antennas have been discussed from an aspect of achieving a compact structure. It is demonstrated that a zigzag dipole, has the absolute gain and the half-power beamwidth comparable to those of a half-wave linear dipole. The size reduction is $24 \%$. In a meander-line dipole, the size reduction of $30 \%$ is obtained. However there was no insight into bandwidth characteristic.

Therefore most popular antennas can be miniaturized using folding and shorting technique. However, this technique comes at a cost of decreasing the bandwidth of the antenna. Moreover, this method complicates the geometry of the antenna which adds to the complexity of the structure and its conformity.

\subsection{Modification of Ground Plane}

Antenna can also be miniaturized by modifying the ground plane, many types of modification can be applied to the ground plane, in this paper we will report four types that are widely used in literature:

\subsubsection{Reduced the Size of the Ground Plane}

Conventional antennas assume infinitely large ground plane dimensions and thus they are large in size. The size of ground plane is the limitation of antenna characteristics and compactness. Since in practice, antennas must have finite ground plane, its effect should be considered in the analysis and design procedures. An MPAs with truncated ground planes have been analyzed analytically in various works [5153].

In [51] John Huang reported on the effect of finite ground plane on the microstrip antenna radiation patterns, he calculate the edge diffracted fields from the finite ground plane of a microstrip antenna to demonstrate that the accuracy of the calculations should take into consideration the effect of the ground plan. Also numerical examples demonstrated that the finite edge calculation is important if accurate pattern levels at wide angles and backlobe information are required. Also it was shown that back lobe radiation could not be neglected in the case of finite ground plane. 
Mohamed Tarbouch, Abdelkebir El Amri and Hanae Terchoune; Contribution to the Miniaturization of Antennas: State of the Art, Transactions on Networks and Communications, Volume 4 No. 5, October (2016); pp:48-77

[52] Reported an analytical technique for the finite ground plane effect on radiation characteristics of a microstrip antenna. Theoretical results show that the gain of a circular patch antenna varies with the ground plane size and is at its maximum when the radius of the ground plane is $0.63 \lambda 0$. It was also found that the input impedance changes widely with the ground plane dimension and decreases with increase in the ground plane radius. The induced current on the ground plane, derived there in, is an approximate one and does not include the edge effects. In order to incorporate the edge effects, a physical theory of diffraction analysis needs to be added. If the ground plane size is not too small, as in the most practical cases, the existing theory gives fairly accurate results for the radiation characteristics. So changing the ground plane size affect directly the input impedance, gain and radiation characteristics of an antenna.

In [53] A set of computed Results shown that when the ground plane size is about the patch size the directivity is minimum. It increases initially by increasing the ground plane size, and exceeds well beyond infinite ground plane directivity. It was found finaly that at size of the ground plane of $1.1 \lambda 0$ ( $\lambda 0$ is the wavelength at resonance frequency) the best directivity can be obtained.

\subsubsection{Insertion of Slots in the Ground Plane}

Along with reducing the ground plane, many other modifications in the ground plane are possible to miniaturize an antenna. These modifications include the insertion of various types of slots in the ground plane. These slots, when properly designed, help to increase the current path within the patch area. This helps in lowering the resonant frequency of the antenna and, therefore, leads to size-reduction. Many designs have appeared in the literature which uses different slots in the ground plane to miniaturize an antenna [54-56].In [54] as shown in Figure21 an annular slot was engraved out in the ground plane underneath the patch for miniaturization. This slot loading resulted in decrease of the resonant frequency of the MPA and it shifted from $2.5 \mathrm{GHz}$ to $1.75 \mathrm{GHz}$. Thus, $52 \%$ reduction in terms of the area of the patch was achieved. Further, the frequency reconfigurable feature is realized by loading the slot with a reactive impedance using the varactor diode.

In[55] a simple procedure is presented to miniaturize an omnidirectional Ultra Wide Band (UWB) planar antenna using pairs of slots in the radiator and the ground plane, the proposed method reduces the antenna's surface area by $50 \%$, with the same bandwidth, efficiency and radiation pattern.

In [56] a new design of a compact microstrip antenna with meandering slots in the antenna's ground plane was proposed, It is found that the proposed design can cause lowering of the antenna's fundamental resonant frequency from $2387 \mathrm{MHz}$ to $1587 \mathrm{MHz}$. This means that the antenna size reduction as large as about $56 \%$ can be achieved for the proposed antenna for operating at a fixed frequency. Moreover, it is seen that the impedance bandwidths and measured antenna gain are greater than that of the refence antenna.

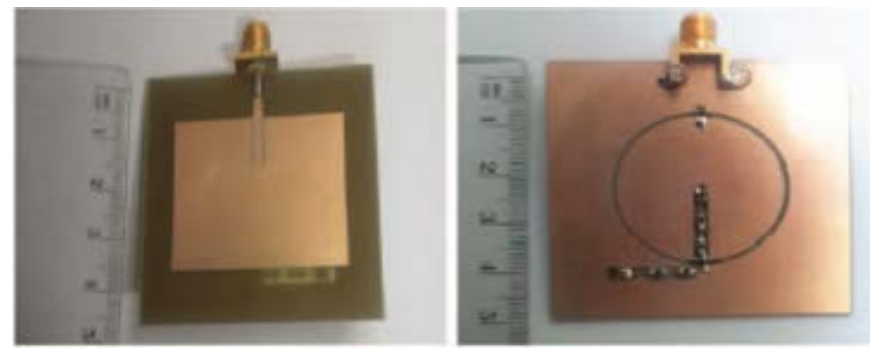

Figure21: Fabricated reconfigurable and miniaturised MPA, (a) Top side, (b) Bottom side 


\subsubsection{Irregular Grounde Strucrure}

Another method which made use of an irregular ground structure was presented in [57-58] for antenna miniaturisation. In [57] a Circularly Polarized Patch Antenna is miniaturized, the paper proposed a novel substrate integrated irregular ground structure by using connected vias which can be easily fabricated to form metallic walls. As shown in Figure22 there are two parallel metallic walls located under each corner of the radiating patch. Capacitive and inductive loadings created by the proposed structure can reduce resonate frequency of antenna efficiently. With this structure, $62 \%$ reduction on resonate frequency was achieved experimentally and the total area of the patch has been reduced by $86 \%$ effectively. In [58] The same technique for size-reduction was used for a linearly polarized MPA, 52\% reduction on resonant frequency was achieved experimentally and the total area of the patch has been reduced by $77 \%$. However, the bandwidth was reduced from 8.3\% (reference antenna with absence of the irregular ground structure) to $1.9 \%$. In the same study a miniaturized CP antenna with $77 \%$ size reduction was also implemented with irregular ground structure technique.

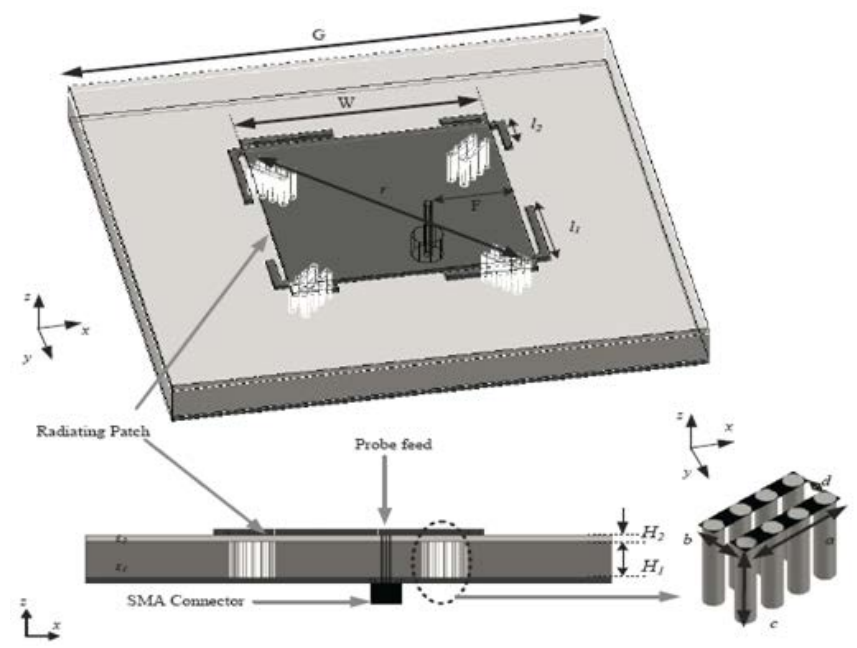

Figure22: A circularly polarized patch antenna with substrate integrated irregular ground plane

\subsubsection{Defected Grounde Strucrure}

Many miniaturized MPA designs use defected ground structures (DGSs). DGSs have a variety of shapes, including simple ones such as spiral, $\mathrm{V}$-shape, U-shape and $\mathrm{H}$-shape, as well as complex ones such as dumbell-shaped and split-ring resonators (SRRs).

Complementary SRR (CSRR)-based antenna miniaturization has been presented in [59-62]. In these Studies, the CSRR has been used at different locations underneath the patch for antenna miniaturisation.

In [59], two slotted CSRRs were engraved out from ground plane, and placed directly underneath the antenna's non radiating edges, as shown in Figure23. The technique is simple and easy to fabricate using traditional milling techniques. It was found that a size reduction of almost $10 \%$ is achieved. The radiation efficiency of the patch antenna with and without SCSRRs shows same performance at the resonant frequencies, which was around $88 \%$.

In [63] a microstrip antenna with dumbbell shaped defected ground structure was proposed, The DGS consists of two rectangular areas and one connecting slot in the ground plane, from The Transmission Line model the antenna size $14.5 \mathrm{mmX} 18.0 \mathrm{mmX} 1.524 \mathrm{~mm}$ is calculated for the frequency $5.2 \mathrm{GHz}$ and is 
optimized, With the dumbbell defect in the ground plane the antenna size reduced to $12 \mathrm{mmX} 15 \mathrm{mmX}$ $1.524 \mathrm{~mm}$ for the same resonance frequency, the size reduction is about $31 \%$. Another method was used to improve the antenna efficiency.

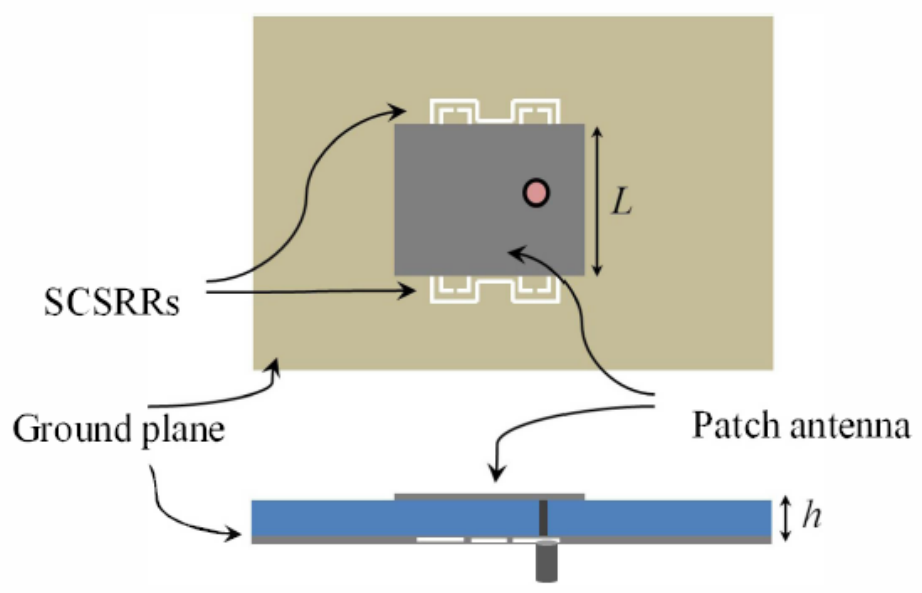

Figure23: View of a square microstrip patch antenna with the resonators Engraved from ground plane under the antenna. The white dashed lines represent the engraved SCSRRs from ground plane

Indeed, the modification of the ground plane can be used for antenna miniaturization. This technique uses various methods starting from simple design to complex one allowing different degree of miniaturization. However as per John Huang conclusion the back lobe radiation could not be neglected in the case of finite ground plane. Also in some cases the antenna should be tunned after ground plan modification in order to improve the bandwidth and the efficiency.

\subsection{Top Loading}

If we consider the monopole above ground shown in Figure24, the current distribution indicated can be maintained by keeping the length of the antenna approximately constant, but having some of the structure located parallel to the ground, as shown for the ' $T$ ', 'Star' and 'Disc' configurations. This allows the height of the antenna to be reduced without changing the resonant frequency.

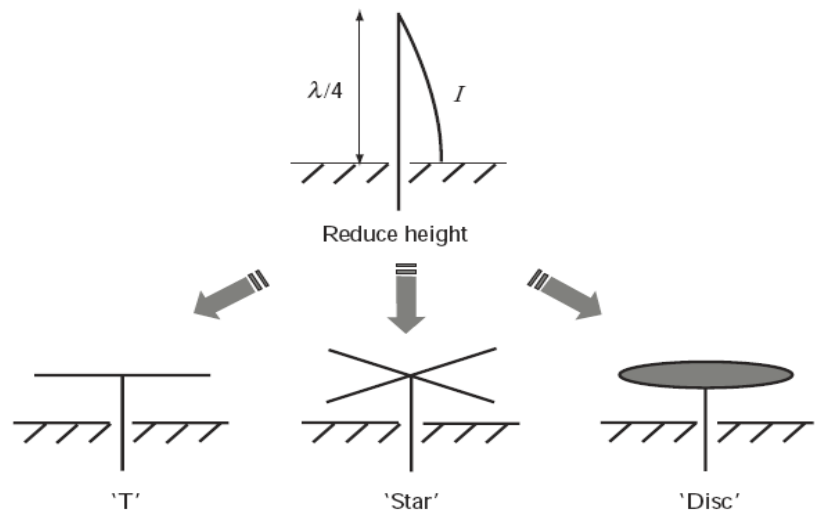

Figure24: Height reduction via top loading

A number of works have appeared in the literature that presented the design or analysis of miniaturized monopole antenna using the disk top loading techniques [64-66]. As shown in Figure25 top loading with a capacitive disk is a way of reducing the antenna height [64], However without folding the vertical 
element, which allows transformation of the input impedance to compensate for the radiation resistance reduction that occurs when the size is decreased, the bandwidth will be decreased due to fundamental limitations on the antenna $Q$. For this reason the proposed antenna used a tuning method of the vertical element to obtain a broad band. The radiation pattern and the radiation efficiency are nearly that of a monopole. The final antenna size compared to conventional one was not highlighted in the paper.

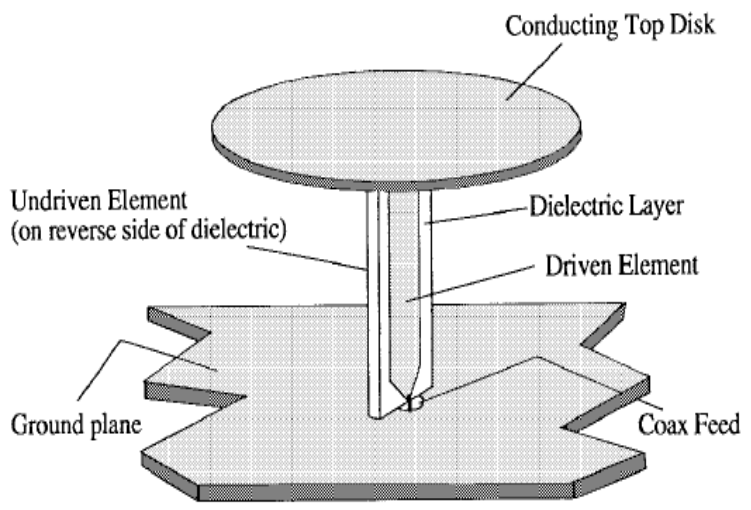

Figure 25: Disk loaded monopole with vertical element formed from two parallel strips separated by a dielectric

In [65] a circular disk top-loaded monopole antenna was proposed, the circular disk is attached on the top of the monopole antenna to miniaturize the antenna. Four metal posts are arranged around the monopole antenna, the metal posts are connected to the circular disk and are shorted to the ground plane. The arrangement of shorting posts mainly serve to widen the antenna operational frequency band. Using this arrangement the antenna height was shifted from $0.25 \lambda$, antenna height of a usual monopole, to $0.1 \lambda 0$. The height of the antenna was, therefore, reduced by $60 \%$. A frequency bandwidth over $18.5 \%$ was achieved with a $-14 \mathrm{~dB}$ return loss (VSWR of 1.5:1).

In [66] as shown in Figure26 a novel 3D miniaturized RFID antenna is proposed. It consists of two vertical printed triangular monopole antenna elements. And top-loading is used to reduce the antenna's profile. Four tuning poles are adopted for better matching and broader bandwidth. The measured return loss is $33 \mathrm{~dB}$ and its $-10 \mathrm{~dB}$ bandwidth is about $20 \%$. The measured maximum gain is $3.73 \mathrm{~dB}$. Compared with conventional RFID antenna, $50 \%$ size reduction is achieved.

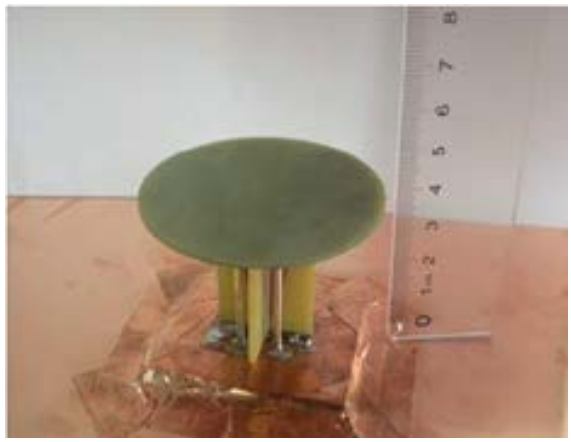

Figure 26 : Photograph of the fabricated antenna

The same technique was used in [67] to present a low profile UWB antenna that covers an operational frequency bandwidth of $120 \%$. The antenna covers frequencies from $700 \mathrm{MHz}$ to $3000 \mathrm{MHz}$. The antenna 
Mohamed Tarbouch, Abdelkebir El Amri and Hanae Terchoune; Contribution to the Miniaturization of Antennas: State of the Art, Transactions on Networks and Communications, Volume 4 No. 5, October (2016); pp:48-77

is constructed from a top loaded monopole with a capacitive metal plate to reduce its height by $35 \%$ and a shorting post and ground sleeve to increase its operational frequency bandwidth from $15 \%$ to $125 \%$.

Also many efforts have been made to reduce antenna size using top loading technique based on wire structures. In [68] Metallic paths patterned according to Peano and Hilbert fractal curves are used as toploads for miniaturization of monopole antennas. In particular, it is shown that a Peano-curve top-loaded monopole results in a matched electrically small antenna with a height of about 0.08 wavelengths and a perfect monopolar radiation pattern. Preliminary experimental results confirm the relatively wideband nature of this miniaturized monopole. In [69] a method to reduce the size of a conventional LPDA (LogPeriodic Dipole Array) is discussed. T-top loaded dipoles are implemented in the array instead of $\lambda / 2$ dipole elements to achieve a reduced size and maintain co-planar geometry. Each dipole in the array is reduced in size by $50 \%$. The simulated S11 of the size reduced LPDA is maintained below $-10 \mathrm{~dB}$ across the conventional LPDA's bandwidth from $35 \mathrm{MHz}$ to $60 \mathrm{MHz}$. The size-reduced LPDA has directive realized gain reaching $9.5 \mathrm{dBi}$. The Figure27 shows the geometry of both the full-sized LPDA and the size-reduced LPDA with T-top loaded dipole elements.

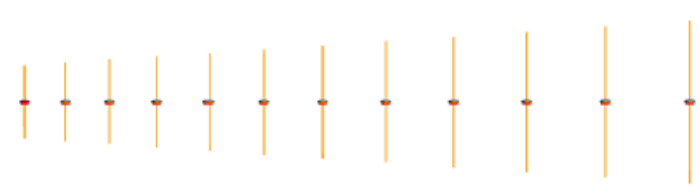

(a) Full-sized LPDA

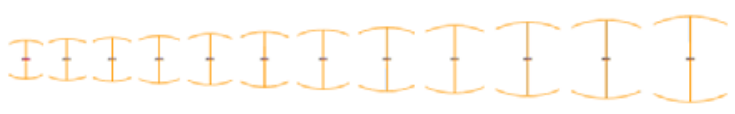

(b) Size-reduced LPDA

Figure27 : Element LPDA Geometry

Therefore miniaturization of antenna can be achieved by top loading technique, the technique is simple and only required adding new structure design in the top of the antenna. Most presented works in this topic signal that final antenna structure should be tunned in order to obtain a broad band. Also It is important to note that while all of the above focused on the miniaturization of antenna, they did not clearly analyze other parameters that are affected by such miniaturization. Therefore, in most of these works, the effect of miniaturization on antenna efficiency, bandwidth, radiation properties, crosspolarization levels etc., was not clearly mentioned and compared with conventional structure.

\subsection{Use of Metamaterials}

Metamaterials (MTMs) are artificially engineered materials designed to provide material properties not readily available commercially. MTMs can be designed to realize materials with near zero values of permittivity; negative permittivity or permeability; or simultaneous negative permittivity and permeability.

Materials may be categorized by their constitutive parameters $\varepsilon$ and $\mu$ according to the diagram shown in Figure28. If both the permittivity and permeability have positive real parts as in the first quadrant of Figure28, as most of the materials in nature do, they will be called "double positive (DPS)" media. In contrast, if both of these quantities are negative, as in the third quadrant of Figure28, they will be called "double-negative (DNG)." The materials with one negative parameter, quadrants two and four, will be 
called "singlenegative (SNG)." If the permittivity is negative, as in the second quadrant, these SNG materials will be called "epsilon negative (ENG)."

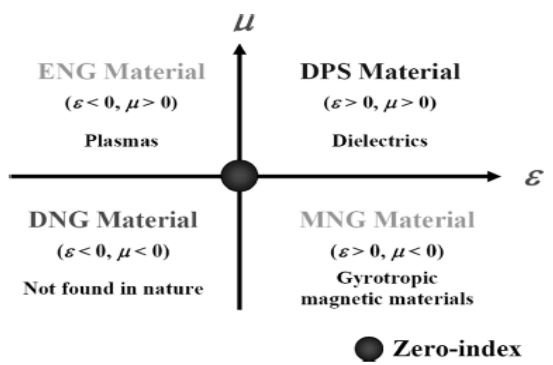

Figure28 : Classification of metamaterials by the real parts of their constitutive parameters, i.e., their permittivity and permeability

In 1967, Viktor Veselago provides the visionary speculation on the existence of MTM "substance with simultaneously negative value of $\varepsilon$ and $\mu$ " [70]. These "substances" LH (left-handed) will allow the propagation of electromagnetic waves with the electric field, the magnetic field, and the phase constant vectors building a left-handed triad, compared with conventional materials where this triad is known to be right-handed [71].

Many works interested to antenna miniaturization using MTMs as a substrate based on ENG, MNG or DNG materials. The proposed MTM substrates were fabricated by loading a host dielectric material by different unit cell shapes.

In [72] an MPA based on magnetic-permeability-enhanced MTM substrate was fabicated. The substrate consisted of periodically spiral loop unit embedded in a host dielectric material, the unit cell was placed vertically between the patch and the ground plane. Compared to conventional MPA printed on an FR4 substrate, this arrangement resulted in significant size reduction of the patch antenna.

The designed metamaterial-substrate antenna achieved a miniaturization factor of approximately 3 in the radiation edge length compared to a rectangular patch antenna operating at the same frequency built on a conventional FR4 substrate. Also, a significant $90 \%$ reduction in the area occupied by the antenna plane was achieved. However, the proposed antenna has a higher thickness than that of a conventional FR4 substrate due to vertically placed unit cell. Concerning others characteristics of designed antenna it was found that the obtained bandwidth is comparable to that of an antenna built on a conventional FR4 substrate. However, compared to conventional based FR4 MPA, the gain was decreased in both azimuth and elevation plane. The measured efficiency for the metamaterial-substrate antenna and FR4 antenna were $30 \%$ and $48 \%$, respectively.

The same technique was used in [73], a circular MPA based on MTM substrate was presented and compared to conventional antenna witch used FR4 substrate. In the new approach, the FR-4 substrate was changed by single split ring unit cell of metamaterial substrate, the single unit cell is shown in Figure29. The new MTM confirmed to act with the negative permittivity equal to -3.09 at resonance frequency. It was found that the new designed MTM substrate was able to reduce size for more than $50 \%$ of the overall circuit area. It is also able to improve the return loss from $-22 \mathrm{~dB}$ to $-24 \mathrm{~dB}$ as well as increase the directivity gain about $1.5 \mathrm{dBi}$ higher than the antenna on FR-4. However both antennas have narrow bandwidth. 


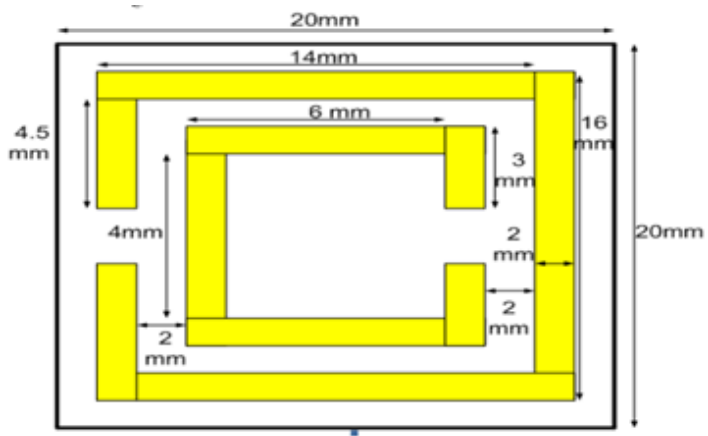

Figure 29 : Dimensions of the single unit cell construction

In [74] two miniaturized dual-band CPW-fed antennas loaded with u-shaped and inverted u-shaped metamaterials unit cells are represented. These antennas consist of a patch and 4-cell unit cells of metamaterial under it, microstrip line, and CPW waveguide. These antennas work at two frequency bands, which at the first band radiates as a monopole antenna with miniaturization of about $35 \%$, radiation efficiency of more than $90 \%$ and improved bandwidth compared to printed monopole antennas and at the second band they radiate as a patch antenna with miniaturization of about $77 \%$ and radiation efficiency of more than $70 \%$ and improved bandwidth compared to conventional patch antennas. Fabricated antennas are shown in Figure30.

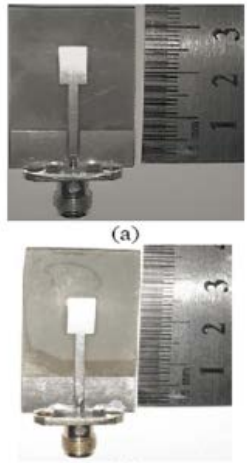

(c)

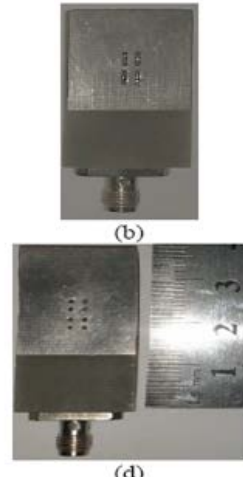

(d)

Figure30 : The built model of the antennas loaded with u-shaped MTM ((a) on surface and (b) bottom) and inverted u-shaped MTM ((c) on surface and (d) bottom).

It is shown in [75] that magnetic resonant structures such as SRRs exhibit mu negative properties only when placed in an environment where the magnetic field is aligned along the SRR axis. By using the concept of duality, it is shown in [76] that epsilon negative properties may be created when a complementary split ring. Resonator (CSRR) is excited by an axial electric field

The principle described above was applied in [77] as shown in Figure31. In this study an MTM-inspired technique was used for circular MPA miniaturization, the methodology consists of horizontal placing of a circular conducting disk a half way between the radiating patch and the ground plane. Optimizations were performed to achieve individual resonances at $4 \mathrm{GHz}, 2 \mathrm{GHz}$ and $0.5 \mathrm{GHz}$. The size reduction at $2 \mathrm{GHz}$ and $0.5 \mathrm{GHz}$ are $60 \%$ and $90 \%$ respectively. The simulated efficiencies at $4 \mathrm{GHz}$ and $2 \mathrm{GHz}$ are $70 \%$ and $51 \%$ respectively. At $500 \mathrm{MHz}$, the efficiency degrades to less than $10 \%$. The unloaded patch antenna was found to be resonant at $5 \mathrm{GHz}$ with an efficiency of $86 \%$. 


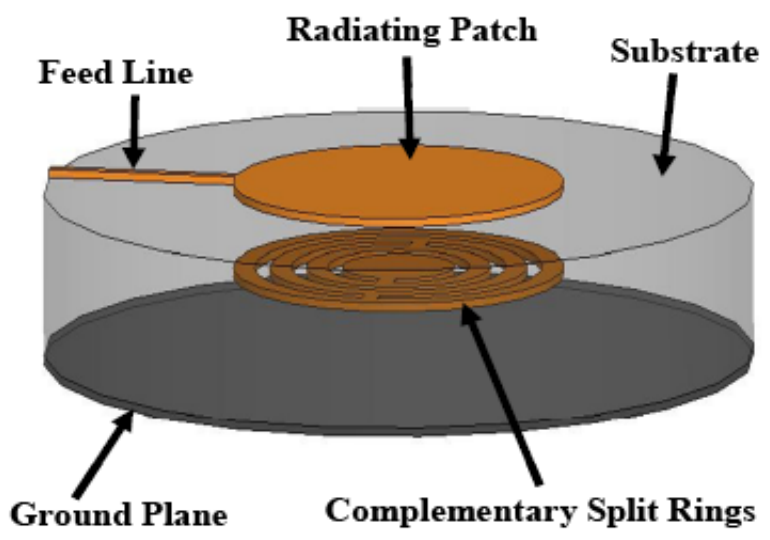

Figure 31: Loaded patch Antenna

The use of MTMs allows obtaining a high degree of miniaturization. However this technique suffers from the complexity of the use of material and in many cases narrow bandwidth or low efficiency are obtained.

\section{Real systems used antenna miniaturization techniques}

In this chapter we present some examples of real systems that used antenna miniaturization techniques.

Example 1: Top loading technique in titanic

An early example of the use of top loading is shown in Figure32. The Titanic famously sank in 1912. Marconi's transmitter equipment was used to sound the alarm using a large ' $T$ ' antenna.

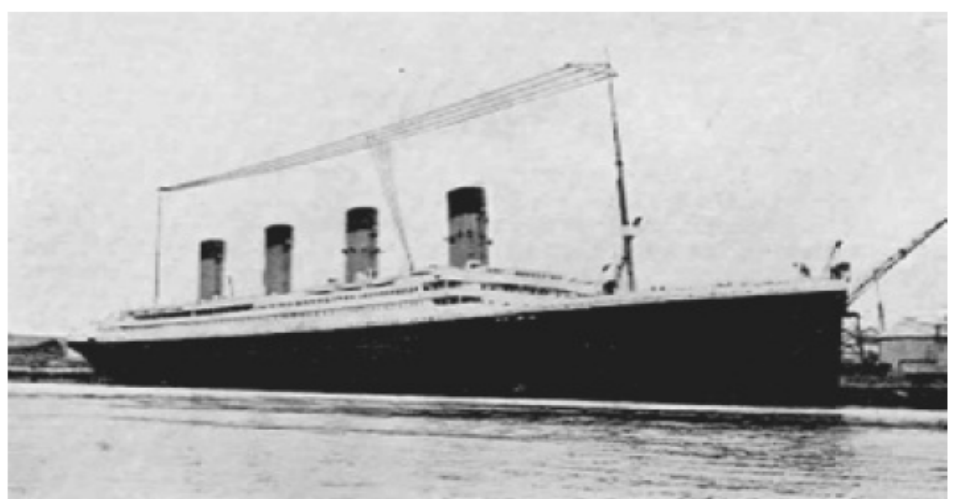

Figure 32: ' $T$ ' Antenna on the Titanic

Example 2: Reactive loading technique in tri-band data modem

The second example is a data modem which uses a tri-band antenna. As shown in Figure33 the antenna uses meandered structure to implement reactive loading technique to cover lower band. 


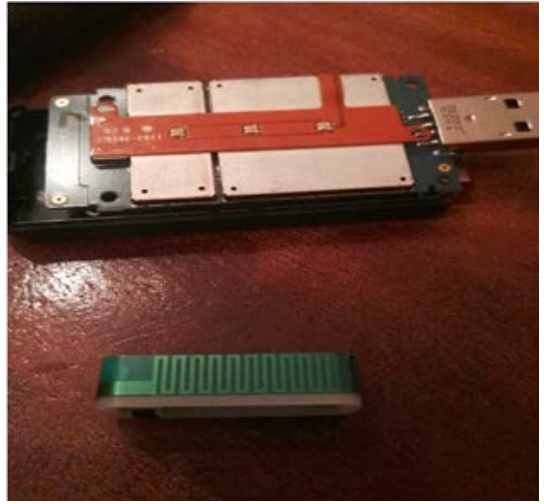

Side view

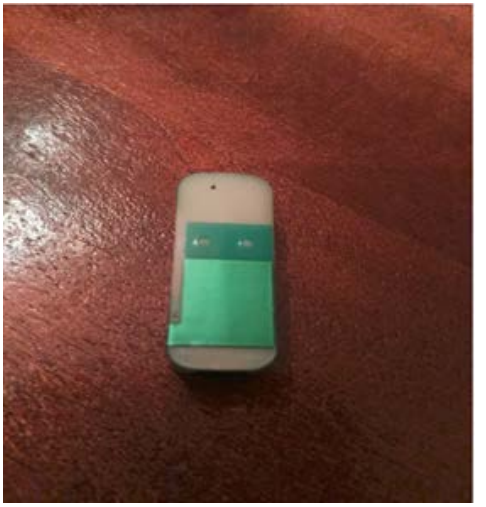

(b) Top view

Figure 33: Dual band antenna using reactive loading technique

\section{Example 3: Shorting technique in smartphones}

Figure34 shown a PIFA (Planar Inverted-F Antenna) implemented in a modern smartphone. As shown in the figure there are two meandered arms, which are essentially both IFAs. The most difficult frequency band to cover is typically the low band, because this is the lowest frequency and requires the most space. Hence, the antenna will first be designed for the low band. Then a smaller arm can be added for the high band (the $1800 \mathrm{MHz}$ cellular frequencies).

The technique used for miniaturization in this smartphone is adding a shorting pin between radiation element and the ground plane to form PIFA antenna.

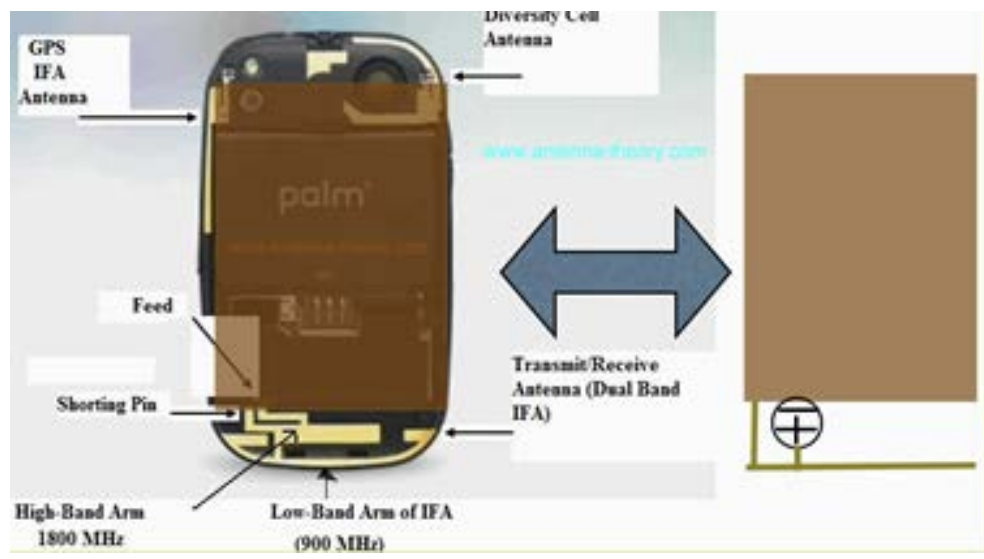

Figure 34: Antenna in smartphone with a simplified diagram

\section{Example 4: Reactive loading technique in Texas instruments usb dongles}

Figure35 shows two $2.4 \mathrm{GHz}$ Texas Instruments USB dongles. The one on the left is an F-antenna compressed to the side. The center device is a straight meander monopole compressed to the end. As shown in reactive loading technique section the mender structure is a way to implement reactive loading technique. 


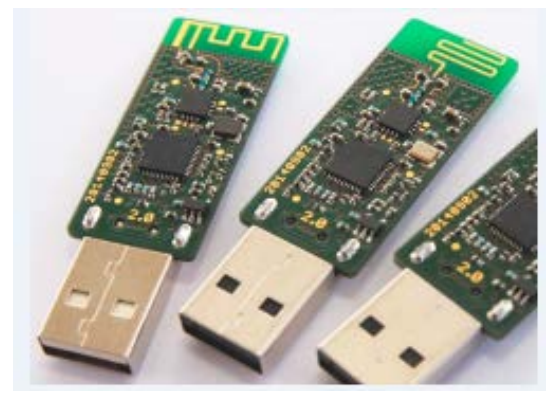

Figure 35: 2.4 GHz Texas Instruments USB dongles

\section{Example 5: Folding technique in tri-band data modem}

In this example (Figure 36) a tri-band data modem operating in GSM 900, GSM 1800 and UMTS using folding monopole to cover the three bands.

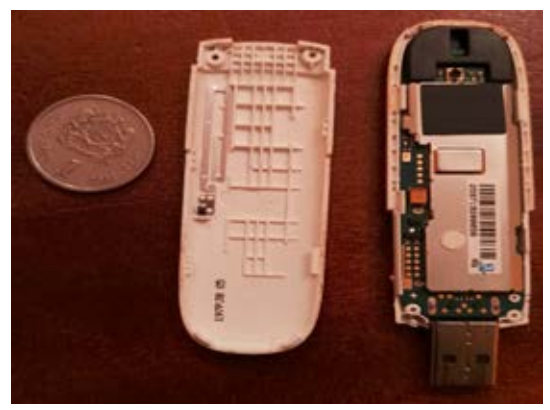

Figure 36: Tri-band data modem antenna

\section{Conclusion}

In this study, we have presented major techniques that have been proposed in the literature for antenna miniaturization. The theory of fundamental limits of antenna size has been presented and it was shown that there are fundamental limits that determine the relationship between antenna size, bandwidth and efficiency. When the antenna size becomes too small, either the bandwidth or the efficiency must be compromised.

The miniaturization techniques that are used in the literature for antenna included reactive loading, material loading, reshaping the antenna geometry, shorting and folding, modification of ground plane, top loading and the use of metamaterials. It was found that some techniques provided high degree of miniaturization, about $90 \%$ of size reduction. Moreover some techniques are simple to be applied and generate simple designs, while others generate complex geometries which are hard to integrate in limited space. Also some techniques need materials that are not available commercially or not low-cost. At the end of this study we presented some examples of real systems that used antennas miniaturization techniques.

Many papers in literature did not clarify the methodology used to design physical antenna geometries, and in many cases they did not present the principles antenna characteristics after miniaturization to conclude about the effect of applied technique to antenna performances.

By presenting each miniaturization technique alone, it would legitimate to ask what would result by combining several techniques simultaneously on the same antenna? Is it possible to optimize the key characteristics of an antenna by application of several techniques simultaneously? 


\section{REFERENCES}

[1] H. Wheeler: 'Fundamental Limitations of Small Antennas', Proc. IRE, December 1947, pp. 1479-1484.

[2] L. J. Chu: 'Physical Limitations of Omni-Directional Antennas', J. Appl. Phys., 19, 1163-1175, 1948.

[3] R. Harrington: 'Effects of Antenna Size on Gain, Bandwidth, and Efficiency', J. Nat. Bur. Stand., 64-D, $1-12,1960$.

[4] R. E. Collin and S. Rothchild: 'Evaluation of Antenna Q', IEEE Trans. AP, AP-12, 23-27, 1964.

[5] R. L. Fante: 'Quality Factor of General Ideal Antennas', IEEE Trans. AP, AP-17, 151-157, 1969.

[6] J. S. McLean: 'The radiative properties of electrically-small antennas', IEEE Int. Sympos. on Electromagnetic Compatibility, 1994, pp. 320-324.

[7] J. S. McLean: 'A re-examination of the fundamental limits on the radiation $Q$ of electrically small antennas' IEEE Trans. AP, 44(5), 672-676, 1996.

[8] K. Konno, H. Wada, and K. Matsukawa: 'Small-sized slot antenna for. ID-car', 1992 Asia Pacific Microwave Conf. Proceedings, vol. 1,1992, pp. 141-144

[9] L. Gil-Young Lee, K. Yonghoon, L. Jong-Sik, N. Sangwook: 'Size reduction of microstrip-fed slot antenna by inductive and capacitive loading', IEEE Antennas and Propagat. Society Int. Symposium ,Vol.1, pp.312-315, 2003

[10] A. K. Shackelford, Sang-Yick Leong, Kai-Fong Lee: 'Simulation of a probe-fed notched patch antenna with a shorting post', IEEE Antennas and Propagation Society International Symposium, pp. 708 - 711 vol.2,2001.

[11] B. Ghosh, SK. M.Haque, D. Mitra and S.Ghosh : 'A Loop Loading Technique for the Miniaturization of Non-planar and Planar Antennas', IEEE Transactions on Antennas \& Propagation, Vol 58, Issue 6, June 2010.

[12] S. H. Kim, J. H. Jang: 'Compact Folded Monopole Antenna with LC-Loadings' Antenna Technology (iWAT), International Workshop, pp. 1-4, 2010

[13] J. A. Tirado-Mendez, H. Jardon-Aguilar, R. Flores-Leal, M. Reyes-Ayala, and F. Iturbide-Sanchez: 'Inductively-Loaded Yagi-Uda Antenna With Cylindrical Cover for Size Reduction at VHF-UHF Bands', IEEE TRANS. ANTENNAS AND PROPAG, VOL. 59, NO. 2, pp. 357-362 Feb 2011

[14] Quan Sun, Jinlong Wang, Jian Cui, Jiahui Fu, Changfei Zhou, Erchao Wang : 'A Compact Printed Logperiodic Antenna with Loaded Stub', Antennas and Propag, 2014 3rd Asia-Pacific Conference pp.593595

[15] M. U. Khan, M. S. Sharawi, and R. Mittra: 'Microstrip Patch Antenna Miniaturization Techniques : A Review' IET Microwaves, Antennas \& Propagation, 2015, Vol. 9, Iss. 9, pp. 913-922 
[16] Kula, J., Psychoudakis, D., Liao, W.-J., Chen, C.-C., Volakis, J., Halloran, J.: 'Patch antenna miniaturization using recently available ceramic substrates', IEEE Antennas Propag. Mag., 2006, 48, (6), pp. 13-20

[17] Ikonen, P.M.T., Rozanov, K.N., Osipov, A.V., Alitalo, P., Tretyakov, S.A.: 'Magnetodielectric substrates in antenna miniaturization: potential andlimitations', IEEE Trans. Antennas Propag., 2006, 54, (11), pp. 3391-3399

[18] Farzami, F., Forooraghi, K.: 'Miniaturization of a microstrip antenna using a compact and thin magneto-dielectric substrate', IEEE Antennas Wirel. Propag. Lett., 2012, 10, pp.1540-1542

[19] N. Venkatarayalu, M. Iddagoda, Ling Bing Kong, Sing-Kwong Ting: 'Reducing the size of monopole antennas using magneto-dielectric material loading', IEEE Antennas and Propagation 2012 ,pp.1-2

[20] Abdelhalim Mohamed, Lotfollah Shafai :' Effect of size reduction on the performance of UWB Vee dipole antennas' Antenna Technology and Applied Electromagnetics IEEE 2012, pp. 1-4

[21] Yifei Shi, Keith W. Whites : 'Miniaturization of helical antennas using dielectric loading', Antennas and Propagation in Wireless Communications IEEE 2014 pp.163-166

[22] Abdelati REHA, Abdelkebir EL AMRI, Othmane BENHMAMMOUCH \& Ahmed OULAD SAID: 'Fractal Antennas: A Novel Miniaturization Technique for wireless Networks', Transactions on Networks and Communications, Volume 2 No 5, Oct (2014), pp. 165-193

[23] N. A. Murad, M. Esa, M. F. M. Yusoff, S. H. A. Ali: 'Hilbert Curve Fractal Antenna for RFID Application’ Proceedings of IEEE International RF and Microwave Conference,Malysia,2006,pp.182-186

[24] Basil K Jeemon, K Shambavi, Zachariah C Alex, : 'A Multi-fractal Antenna for WLAN and WiMAX application', Proceedings of 2013 IEEE Conference on Information and Communication Technologies (ICT 2013).

[25] Ananth Sundaram, 'Koch-Fractal Folded Slot Antenna Characteristics', IEEE Antennas and Wireless Propagation Letters, pp. 219-222, 2007

[26] N. A. Murad: 'Fractal Patch Antenna for GPS Application', Proc. IEEE Student Conf. R\&D, pp. 102-104, 2003

[27] Kakoyiannis, C.G., Constantinou, P.: 'A compact microstrip antenna with tapered peripheral slits for CubeSat RF payloads at $436 \mathrm{MHz}$ : 'miniaturization techniques, design, and numerical results'. IEEE Int. Workshop on Satellite and Space Communications (IWSSC08), October 2008, pp. 255-259

[28] Anguera, J., Boada, L., Puente, C., Borja, C., Soler, J.: 'Stacked H-shaped microstrip patch antenna', IEEE Trans. Antennas Propag., 2004, 52, (4), pp. 983-993

[29] Bokhari, S.A., Zurcher, J.-F., Mosig, J.R., Gardiol, F.E.: 'A small microstrip patch antenna with a convenient tuning option', IEEE Trans. Antennas Propag., 1996, 44, (11), pp. 1521-1528

[30] Chatterjee, S., Ghosh, K., Paul, J., Chowdhury, S.K., Chanda, D., Sarkar, P.P.: 'Compact microstrip antenna for mobile communication', Microw. Opt. Technol. Lett., 2013, 55, (5), pp. 954-957 
Mohamed Tarbouch, Abdelkebir El Amri and Hanae Terchoune; Contribution to the Miniaturization of Antennas: State of the Art, Transactions on Networks and Communications, Volume 4 No. 5, October (2016); pp: 48-77

[31] Chen, W.-S., Wu, C.-K., Wong, K.-I.: 'Square-ring microstrip antenna with a cross strip for compact circular polarization operation', IEEE Trans. Antennas Propag., 1999, 47, (10), pp. 1566-1568

[32] Shackelford, A.K., Lee, K.-f., Luk, K.M.: 'Design of small-size wide-bandwidth microstrip-patch antennas', IEEE Antennas Propag. Mag., 2003, 45, (1), pp. 75-83

[33] Iwasaki, H.: 'A circularly polarized small-size microstrip antenna with a cross slot', IEEE Trans. Antennas Propag., 1996, 44, (10), pp. 1399-1401

[34] Tirado-Mendez, J.A., Jardon-Aguilar, H., Iturbide-Sanchez, F.: 'Application of the defected microstrip structure as a tuning technique for rectangular printed antennas', Micow. Opt. Technol. Lett., 2006, 48, (2),pp. 16-19

[35] Dey, S., Mittra, R.: 'Compact microstrip patch antenna', Microw. Opt. Technol. Lett., 1997, 13, (1), pp. 12-14

[36] Zhang, X.-X., Yang, F.: 'Study of a slit cut on a microstrip antenna and its applications', Microw. Opt. Technol. Lett., 1998, 18, (4), pp. 297-300

[37] Noghanian, S., Shafai, L.: 'Microstrip patch miniaturization by slots loading'. 2005 IEEE Antennas and Propagation Society Int. Symp., 2005, vol. 1B, pp. 215-218

[38] B. Oueslati, T. Ben Salah ; C. L. Aguili ; T. Aguili: 'Patch antenna Miniaturization by inserting of notches and nicks methods'Computer Applications and Information Systems (WCCAIS), 2014 ,pp.1-6

[39] T. Su, Q. Zhang ; R. Chen ; C. Sun:' Novel Design of Surface-Wave Holographic Antenna Miniaturization' IEEE ANTENNAS AND WIRELESS PROPAGATION LETTERS, VOL. 14, 2015, pp. 1077 1080

[40] Abdelati Reha, Marouane BOUCHOUIRBAT : 'A Dual-Band Rectangular CPW Folded SlotAntenna for GNSS Applications' International Journal of Advanced Research in Electrical, Electronics and Instrumentation Engineering,Vol. 3, Issue 8, August 2014

[41] A. M. Abbosh: 'Miniaturized Microstrip-Fed Tapered-Slot Antenna With Ultra Wideband Performance', IEEE Antennas and wireless propagation letters, vol. 8, pp. 690-691, 2009

[42] N. Herscovici, M. Osorio, C. Peixeiro: 'Minimization of a rectangular patch using genetic algorithms' Antennas and Propagation Society International Symposium IEEE, vol 4, pp. 34 - 37, 2001

[43] L. Wakrim, S. Ibnyaich ,M. M. Hassani: 'Optimization by genetic algorithm of a PIFA antenna parameters for Wifi application' IEEE Multimedia Computing and Systems International Conference pp.1501 - 1505,2014

[44] R.B Waterhouse: 'Small microstrip patch antenna', Electron lett, vol. 31, pp. 604-605, 1995

[45] A. Holub, M. Polivka: 'A novel microstrip patch antenna miniaturization technique: a meanderly folded shorted-patch antenna', Microwave Techniques, 2008, COMITE 2008, 14th Conference on, pp: $1-4$. 
[46] Li, R., Dejean, G., Tentzeris, M.M., Laskar, J.: ‘Development and analysis of a folded shorted-patch antenna with reduced size', IEEE Trans. Antennas Propag., 2004, 52, (2), pp. 555-562

[47] J.Rabemanantsoa, Ala Sharaiha: 'Small-folded, printed quadrifilar helix antenna for GPS applications',14th international symposium on Antenna Technology and Applied Electromagnetics ANTEM- 2010, 5-8 July.

[48] R.C. Fenwick: 'A new class of electrically Small antennas', IEEE Trans. on Antennas and Propag., vol. 13, pp. 379-383, 1965.

[49] J. Rashed and C. Tai: 'A new class of resonant antennas', IEEETrans. on Antennas and Propag., vol. 39, pp. 1028-1031,1991.

[50] H. Nakano, H. Tagami, A. Yoshizawa, and J. Yanauchi: 'Shortening ratios of modified dipoles', IEEE Trans. Antennas Propagat., vol. AP-32, pp. 385-386, Apr. 1984.

[51] John Huang : 'The Finite Ground Plane Effect on the Microstrip Antenna Radiation

[52] Patterns', IEEE Transactions on Antennas and Propagation, 31(4), 1983, 649-653.

[53] A. K. Bhattacharyya : 'Effects of finite ground plane on the radiation characteristics of

[54] a circular patch antenna' : IEEE Transactions on Antennas and Propagation, 38(2),1990, $152-159$.

[55] P. Moosavi and L. Shafai: 'Directivity of microstrip square ring antenna and effects of finite ground plane on the radiation pattern parameters', IEEE AP-S Int. Symp. Digest, pp. 672-675, 1998

[56] M. U. Khan, R. Hussain,M. S. Sharawi: 'A compact reconfigurable and miniaturized patch antenna' IEEE 4th Asia-Pacific Conference on Antennas and Propagation (APCAP),pp. 140-141, 2015

[57] A. Abbosh, M. Bialkowski : 'Miniaturization of Ultra-wideband Planar Antenna Using Pairs of Slots in the Radiator and the Ground Plane', IEEE Asia- Pacific Microwave Conference APMC, pp.1-4, 2008.

[58] K. L. Wong, J. S. Kuo and T. W. Chiou: 'Compact Microstrip Antennas with Slots Loaded in the Ground Plane', Antennas and Propagation Eleventh International Conference, vol. 2, pp. 623-626

[59] D. Wang, H. Wong and C. H. Chan: 'Miniaturized circularly polarized patch antenna by substrate integrated irregular ground', Proc. 2011 IEEE AP-S Int. Symp. Antennas Propag., pp. 1875-1878, 2011

[60] Wang, D., Wong, H., Chan, C.H.: 'Small patch antennas incorporated with a substrate integrated irregular ground', IEEE Trans. Antennas Propag., 2012, 60, (7), pp. 3096-3103

[61] Bait-Suwailam, M.M., Al-Rizzo, H.M.: 'Size reduction of microstrip patch antennas using slotted complementary split-ring resonators'. 2013 The Int. Conf. on Technological Advances in Electrical, Electronics and Computer Engineering (TAEECE), May 2013, pp. 528-531

[62] Sharawi, M., Khan, M., Numan, A., Aloi, D.: 'A CSRR loaded MIMO antenna system for ISM band operation', IEEE Trans. Antennas Propag., 2013, 61, (8), pp. 4265-4274 
Mohamed Tarbouch, Abdelkebir El Amri and Hanae Terchoune; Contribution to the Miniaturization of Antennas:

State of the Art, Transactions on Networks and Communications, Volume 4 No. 5, October (2016); pp:48-77

[63] Jang, H.A., Kim, D.O., Kim, C.Y.: 'Size reduction of patch antenna array using CSRRs loaded ground plane'. Progress in Electromagnetics Research Symp., KL, Malaysia, 2012, pp. 14871489

[64] Goodwil, K., Saxena, V.N., Kartikeyan, M.V.: 'Dual band CSSRR inspired microstrip patch antenna for enhancing antenna performance and size reduction'. 2013 Int. Conf. on Signal Processing and Communications (ICSC), 2013, pp. 495-497

[65] A. K. Arya , M. V. Kartikeyan and A. Patnaik: 'Efficiency enhancement of microstrip patch antenna with defected ground structure', Proc. Int. Conf. Recent Adv. Microw. Theory Appl., pp. 729-731, 2008

[66] H. D. Foltz, J. S. McLean, G. Crook: 'Disk-loaded monopoles with parallel strip elements', IET Microwaves, Antennas and Propagation, vol. 1, issue 2, pp. 536-541, April 2007.

[67] T. Noro, Y. Kazama: 'Low Profile and Wide bandwidth Characteristics of Top Loaded Monopole Antenna with Shorting Post', Antenna Technology Small and Novel Metamaterials, pp. 108-111, March 6-8, 2006.

[68] L. Tian, H. Guo, X. Liu, Y.Wang, and X. Yang: 'Novel 3d rfid antenna with low profile and low cost', in Antennas, Propagation EM Theory (ISAPE), 2012 10th International Symposium on, 2012, pp. 69-72.

[69] E. Ghafari, D. N. Aloi: 'Top-loaded UWB monopole antenna for automotive applications', Antennas and Propagation, IEEE, pp.1-2, 2012

[70] J. McVay and A. Hoorfar: 'Miniaturization of top-loaded monopole antennas using Peano-curves', IEEE Radio and Wireless Symp., pp. 253-256, 2007

[71] D. S. Rhodes and S. Lim: 'Size reduction of the log-periodic dipole array', Proc. IEEE Antennas Propag. Soc. Int. Symp., pp. 1578-1579, 2013

[72] V. Veselago: 'The electrodynamics of substances with simultaneously negative values of $\varepsilon$ and $\mu$ ', Soviet Physics Uspekhi, vol. 10, no. 4, pp. 509-514, Jan., Feb. 1968.

[73] Caloz and T. Itoh, Electromagnetic Metamaterials: 'Transmission Line Theory and Microwave Applications', Piscataway, NJ: Wiley- IEEE, 2005.

[74] Mookiah, P., Dandekar, K.: 'Metamaterial-substrate antenna array for MIMO communication system', IEEE Transactions on Antennas and Propagation, 2009, 57, (10), pp. 3283-3292

[75] M. Z. M. Zani, M. H. Jusoh, A. A. Sulaiman, N. H. Baba, R. A. Awang and M. F. Ain: 'Circular Patch Antenna on Metamaterial, Electronic Devices', 2010 International Conference on Systems and Applications (ICEDSA), pp. 313-316

[76] Seyed Amir Hossein Saghanezhad and Zahra Atlasbaf: 'Miniaturized Dual-Band CPW-Fed Antennas Loaded With U-Shaped Metamaterials', IEEE Antennas and Wireless Propagation Letters, vol. 14, pp. $658-661,2015$ 
[77] J. B. Pendry, A. J. Holden, D. J. Robbins, and W. J. Stewart: 'Magnetism from conductors and enhanced nonlinear phenomena', IEEE Trans. Microw. Theory Tech., vol. 47, no. 11, pp. 2075-2084, Nov. 1999.

[78] F. Falcone, T. Lopetegi, J. D. Baena, R. Marqués, F. Martín, and M. Sorolla: 'Effective negative-stopband microstrip lines based on complementary split ring resonators', IEEE Microw. Wireless Compon. Lett., vol. 14, no. 6, pp. 280-282, Jun. 2004.

[79] R. Ouedraogo and E. Rothwell: 'Metamaterial Inspired Patch Antenna Miniaturization Technique', IEEE International Symposium on Antennas and Propagation Digest, pp. 1-4, Jul.2010.

[80] Yi Huang, Kevin Boyle: 'Antennas from theory to practice'. Library of Congress Cataloging-inPublication Data, 2008 edition. 